Supplement 1,

Band 23,

März 2007

Rudolf A. Weiner,

Frankfurt/M.

(Hrsg.)

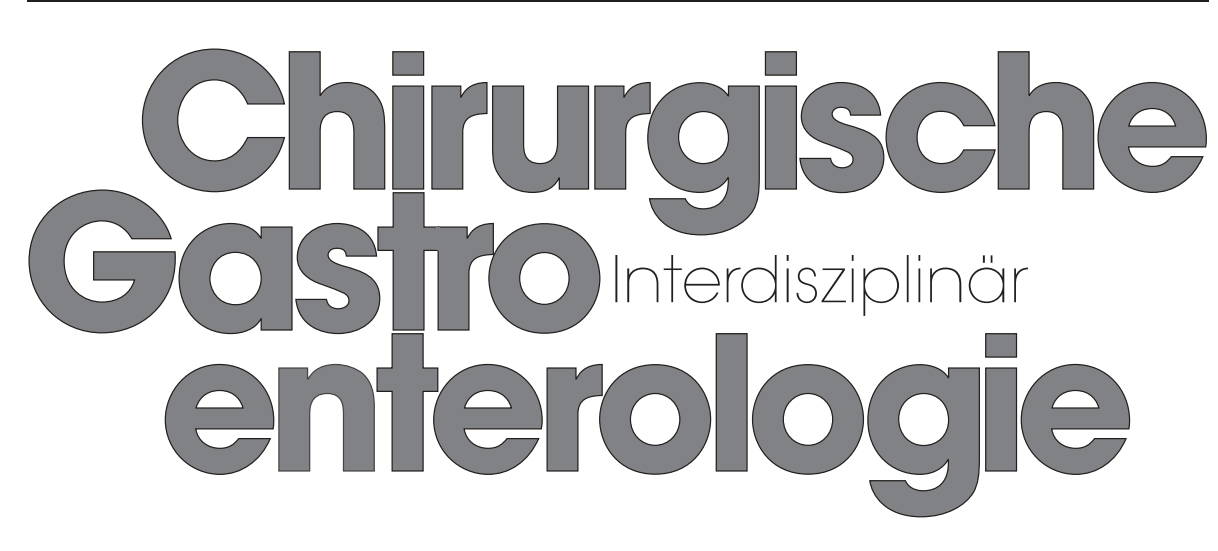

Adipositas-Chirurgie

4. Frankfurter Meeting

Frankfurt/M., 9./10. November 2006 


\section{Band 23, Supplement 1, März 2007}

Editorial

1 Die Zukunft der Adipositas-Chirurgie in Deutschland Weiner, R.A. (Frankfurt/M.)

Übersichtsarbeiten

4 Ergebnisse einer deutschlandweiten Umfrage Komplikationen nach steuerbarem Magenband Stroh, C.; Manger, T. (Gera)

9 LaparoskopischesMagenband bei Patienten mit einem BMI über $60 \mathrm{~kg} / \mathrm{m}^{2}$ - macht es Sinn?

Hesse, U.J.; Gärtner, D.; Münz, K.; Hornung, A. (Stuttgart)

13 10-Jahres-Ergebnisse mit dem Magenband:

Sind Bandversager Folge eines Fehlers?

Stroh, C.; Hohmann, U.; Schramm, H.; Manger, T. (Gera)

19 Laparoskopisches Magenband: Bandmigration was nun?

Tigges, W.; Pick, P. (Hamburg)

21 Prinzip des proximalen Magen-Bypasses Weiner, R.A. (Frankfurt/M.)

30 Potentielle Mangelerscheinungen und Supplementation nach Magen-Bypass

Ludwig, K.; Bernhardt, J. (Rostock)

Originalarbeit

35 Biliopankreatische Diversion mit Duodenal-Switch: Verfahren nach Bandversagen

Manger, T.; Hohmann, U.; Stroh, C. (Jena)

Übersichtsarbeiten

39 Die Notwendigkeit einer interdisziplinären Nachsorge nach bariatrischen Verfahren aus psychologischer Sicht Lager, C.; Schowalter, M.; Bueter, M.; Maroske, J.; Thalheimer, A. (Würzburg); Benecke, A. (Mainz); Fein, M.; Faller, H. (Würzburg)

44 Prinzipien der Supplementation nach Adipositaschirurgischen Maßnahmen Wolf, A.M. (Ulm)

48 Adipositas-Chirurgie und Diabetes Rosak, C.; Weiner, R.A. (Frankfurt/M.)

\section{Vol. 23, Supplement 1, March 2007}

Editorial

1 The Future of Obesity Surgery in Germany Weiner, R.A. (Frankfurt/M.)

Review Articles

4 Results of an Inquiry in Germany - Complications after Adjustable Gastric Banding

Stroh, C.; Manger, T. (Gera)

9 Laparoscopic Gastric Banding in Patients with a BMI over $60 \mathrm{~kg} / \mathrm{m}^{2}$ - Does It Make Sense?

Hesse, U.J.; Gärtner, D.; Münz, K.; Hornung, A. (Stuttgart)

13 10-Year Long-Term Results after Gastric Banding:

Are Failures the Result of a Mistake?

Stroh, C.; Hohmann, U.; Schramm, H.; Manger, T. (Gera)

19 Laparoscopic Gastric Banding: Migration of Gastric Banding What to Do?

Tigges, W.; Pick, P. (Hamburg)

21 Principle of the Proximal Gastric Bypass Weiner, R.A. (Frankfurt/M.)

30 Potential Nutritional Deficiencies and Supplementation following Gastric Bypass Surgery

Ludwig, K.; Bernhardt, J. (Rostock)

Original Article

35 Biliopancreatic Diversion with Duodenal Switch: A Procedure after Failed Gastric Banding Manger, T.; Hohmann, U.; Stroh, C. (Jena)

Review Articles

39 The Need of an Interdisciplinary Follow-Up after Bariatric Surgery from a Psychological Perspective

Lager, C.; Schowalter, M.; Bueter, M.; Maroske, J.; Thalheimer, A.

(Würzburg); Benecke, A. (Mainz); Fein, M.; Faller, H. (Würzburg)

44 Principles of Supplementation after Bariatric

Surgery

Wolf, A.M. (Ulm)

48 Obesity Surgery and Diabetes Rosak, C.; Weiner, R.A. (Frankfurt/M.)

\footnotetext{
KARGER ๑ 2007 S. Karger GmbH, Freiburg 


\section{Band 23, Supplement 1, März 2007}

52 Lebensqualität nach Adipositas-Chirurgie: Ergebnisse nach verschiedenen Operationsverfahren - erste Ergebnisse einer prospektiven Längsschnittstudie

Weiner, S.; Weiner, R.A. (Frankfurt/M.); Rosenthal, A. (Würzburg); Pomhoff, I. (Frankfurt/M.)

Originalarbeit

55 Studie zur Qualitätskontrolle der operativen Therapie der Adipositas - Ergebnisse der Pilotphase 2005

Stroh, C. (Gera); Flade-Kuthe, R. (Hannover); Herbig, B. (Hamburg); Höhne, S. (Burlengenfeld); Köhler, H. (Braunschweig); Pick, P.

(Hamburg); Horbach, T. (Erlangen); Weiner, R. (Frankfurt/M.); Wolf, S. (Magdeburg); Wolf, A.M. (Ulm)

II Impressum

U3 Hinweise für Autoren (3. Umschlagseite)

\section{Vol. 23, Supplement 1, March 2007}

52 Quality of Life after Bariatric Surgery: Results of Different Surgical Procedures - First Results of a Prospective Longitudinal Study

Weiner, S.; Weiner, R.A. (Frankfurt/M.); Rosenthal, A. (Würzburg); Pomhoff, I. (Frankfurt/M.)

Original Article

55 Quality Assurance in Bariatric Surgery in Germany Results of the First Year of German Multicenter Trial 2005 Stroh, C. (Gera); Flade-Kuthe, R. (Hannover); Herbig, B. (Hamburg); Höhne, S. (Burlengenfeld); Köhler, H. (Braunschweig); Pick, P. (Hamburg); Horbach, T. (Erlangen); Weiner, R. (Frankfurt/M.); Wolf, S. (Magdeburg); Wolf, A.M. (Ulm)

II Imprint

C3 Guidelines for Authors (Inside back cover) 